\section{Characteristics of Risk-Factor Profiles Associated with Stroke in Patients with Myotonic Dystrophy Type 1}

\section{Abstract}

Objective: Myotonic dystrophy type 1 (DM1) is a rare autosomal dominant disorder with highly variable phenotypic expression. Some patients have diabetes mellitus, dyslipidemia, and/or arrhythmias, which are risk factors for stroke. However, the mechanism of stroke is poorly understood in patients with DM1. We studied the characteristics of risk-factor profiles for stroke associated with DM1.

Patients and methods: We studied 77 patients with DM1 (45 men and 32 women) on the basis of the patients' clinical histories and laboratory and genetic examination results.

Results: The analysis showed that 26 patients (34\%) had dyslipidemia, and $16(21 \%)$ had diabetes. Arrhythmias were diagnosed in 46 patients (61\%), including 11 (14\%) with atrial fibrillation and $9(12 \%)$ with conduction defects. Echocardiographic abnormalities were found in 28 patients (37\%). Eight patients $(11 \%)$ met the criteria for metabolic syndrome. We identified 2 patients $(2.6 \%)$ with ischemic stroke caused by cardiogenic embolism among 77 patients with DM1. One had paroxysmal atrial fibrillation and sick sinus syndrome, and the other had cardiac dysfunction with an ejection-fraction of $35 \%$ and dyslipidemia. Both patients had highly expanded numbers of CTG repeats (1000 and 1500).

Conclusion: To our knowledge, this is the first study to report a comprehensive analysis of risk-factor profiles for stroke in patients with DM1. Stroke is a relatively rare, but severe complication of DM1. Our results indicate that it is important to manage risk factors for stroke, especially cardiac involvement and arrhythmias.

Keywords: Myotonic dystrophy; Muscular dystrophy; Myopathy; Stroke; Arrhythmias; Atrial fibrillation; Dyslipidemia
Miho Sugie ${ }^{1,2}$, Kazuma Sugie ${ }^{2}$, Nobuyuki Eura², Naoki lwasa ${ }^{2}$, Tomo Shiota ${ }^{2}$, Hitoki Nanaura², Tesseki Izumi ${ }^{2}$ and Satoshi Ueno ${ }^{2}$

1 The Department of Neurology, Nara Prefectural General Rehabilitation Center, Shiki, Nara, Japan

2 The Department of Neurology, Nara Medical University School of Medicine, Kashihara, Nara, Japan

Corresponding author: Sugie K

” ksugie@naramed-u.ac.jp

Department of Neurology, Nara Medical University School of Medicine, 840 Shijocho, Kashihara, Nara 634-8522.

Tel: +81-744-29-8860

Fax: +81-744-24-6065

Citation: Sugie M, Sugie K, Eura N, et al. Characteristics of Risk-Factor Profiles Associated with Stroke in Patients with Myotonic Dystrophy Type 1. J Rare Disord Diagn Ther. 2016, 2:4.

\section{Abbreviations}

DM1: Myotonic Dystrophy Type 1; MRI: Magnetic Resonance Imaging; HDL: High-Density Lipoprotein; NIHSS: National Institutes of Health Stroke Scale; MCA: Middle Cerebral Artery; SD: Standard Deviation; MRA: Magnetic Resonance Angiography

\section{Introduction}

Myotonic dystrophy type 1 (DM1) is a rare neuromuscular disorder with an incidence of approximately 1:15.000 and the most common type of adult muscular dystrophy [1-5]. DM1 is an autosomal dominant disorder with highly variable phenotypic expression. The genetic basis of DM1 is an abnormal expansion of a CTG trinucleotide repeat in the 3'-untranslated region of a protein kinase (DMPK) gene on chromosome 19q21.3 [6].

DM1 is a multisystem disorder associated with myotonia, progressive distal weakness/atrophy of skeletal muscles, cataracts, and systemic manifestations. In fact, DM1 is often complicated by dyslipidemia, arrhythmias, or abnormal glucose tolerance which is risk factors for stroke [1-5]. However, the 
pathomechanism of stroke in patients with DM1 has not been well established or assessed.

This study was designed to assess the characteristics of the riskfactor profiles associated with stroke in patients with DM1, with the ultimate goal of providing suggestions for ways to improve disease management.

\section{Patients and Methods}

\section{Patients}

The study was conducted between April 1, 2005 and March 30, 2014 in the Department of Neurology, Nara Medical University. Seventy-seven Japanese patients with DM1 (45 men and 32 women, mean age 51 years) were consecutively enrolled. Patients younger than 18 years were excluded from this study. We also excluded patients with congenital forms of DM1, since they have been shown to have a different phenotype. Diagnosis of DM1 was attested by the results of clinical and electrophysiological examinations. All patients showed evidence of degenerative myopathy on clinical examinations as well as myotonia on clinical or electromyographic examinations, or both. Neurologically, the patients were examined and evaluated by at least three neurologists. In this study, grip myotonia was defined as delayed ability to relax the muscles after making a forceful grip.

We evaluated the characteristics of various risk-factor profiles associated with stroke on the basis of the patients' clinical histories and laboratory examination results. In accordance with current guidelines of the American Stroke Association, risk factors were classified with respect to their strength of evidence and potential for modification [7]. Dyslipidemia, diabetes mellitus, and hypertension are well documented and modifiable risk factors for stroke. Cardiogenic involvement such as atrial fibrillation is an important risk factor for ischemic stroke. We also assessed D-dimer levels, cigarette smoking, and alcohol consumption, which are potentially modifiable risk factors for stroke, and performed electrocardiography and echocardiography. In addition, we assessed signs and consequences of atherosclerosis by brain Magnetic Resonance Imaging (MRI) and carotid artery ultrasonography. Moreover, the clinical and neuroradiological features of patients who had a previous history of stroke were analyzed. We also considered associations between the main cause of stroke and risk factors for stroke, respectively.

Furthermore, we assessed the role of metabolic syndrome. The criteria for metabolic syndrome were based on the joint statement of the International Diabetes Federation Task Force on Epidemiology and Prevention, National Heart, Lung, and Blood Institute, American Heart Association, World Heart Federation, International Atherosclerosis Society, and International Association for the Study of Obesity [8]. A diagnosis of metabolic syndrome requires the presence of at least 3 of the following 5 factors: central obesity (in Japanese: male waistline $\geq 85 \mathrm{~cm}$, female waistline $\geq 90 \mathrm{~cm}$ ), hypertriglyceridemia $(\geq 150 \mathrm{mg} / \mathrm{dL}$ ), a low High-Density Lipoprotein (HDL) cholesterol level ( $<40 \mathrm{mg} / \mathrm{dL}$ ), hypertension (systolic blood pressure $\geq 130 \mathrm{mmHg}$ or diastolic blood pressure $\geq 85 \mathrm{mmHg}$ ), and diabetes mellitus (fasting blood glucose $\geq 100 \mathrm{mg} / \mathrm{dL}$ ).

\section{Statistics}

Statistical analysis was performed with the use of Statcel 3 (OMS, Inc., Tokorozawa, Saitama, Japan). Data are expressed as the means \pm 1 standard deviation. $P$ values of $<0.05$ were considered to indicate statistical significance. All patients gave fully informed consent before participation. This study was approved by the Ethics Committee of the Nara Medical University School of Medicine.

\section{Genetic analysis}

Genetic studies of the expansion of CTG repeat length in the 3 untranslated regions of myotonin protein kinase were performed with the use of DNA obtained from leukocytes in 50 patients in this study. Digestion with Eco $\mathrm{RI}$ and $\mathrm{Bgl}$ I restriction enzymes, separation of DNA fragment by gel electrophoresis, transfer of the DNA fragment to a nylon membrane by Southern blotting, hybridization with radiolabeled DM1-specific probe, and finally visualization by autoradiography were performed [6].

\section{Results}

\section{Clinical findings}

A total of 77 patients with DM1 were enrolled. Patients were predominantly male (58\%), with a mean age of $51 \pm 15$ years (range 24-84 years) and mean disease duration of $15 \pm 12.1$ years. The sociodemographic and clinical characteristics of the 77 patients with myotonic dystrophy type 1 are presented in Table 1.

Among all enrolled patients with DM1, 26 (34\%) had dyslipidemia, including hypertriglyceridemia in 23 (30\%) and low HDL cholesterol levels in 9 (12\%); 16 (21\%) had diabetes mellitus, and $8(11 \%)$ had hypertension. Arrhythmias were diagnosed in 46 patients $(61 \%)$, including $11(14 \%)$ with atrial fibrillation, 9 (12\%) with conduction defects, and 5 (6.5\%) with atrial flutter. Echocardiographic abnormalities were found in 28 patients (37\%). A pacemaker was implanted in 4 patients $(5.2 \%)$. On neuroimaging including brain MRI, carotid ultrasonography, or both, 19 patients ( $25 \%$ ) showed atherosclerosis of the intracranial arteries. In addition, 10 patients (13\%) had high D-dimer levels. No patient in our series had anti-phospholipid syndrome, protein $\mathrm{S}$ deficiency, protein $\mathrm{C}$ deficiency, or hyperhomocysteinemia.

Eight patients (11\%) met the criteria for metabolic syndrome. The most common component in the patients with DM1 was hypertriglyceridemia (30\%), followed by central obesity, hyperglycemia, and low HDL levels; hypertension (11\%) was the rarest component. Metabolic syndrome was not associated with age, disease duration, or the number of CTG repeats.

We identified 2 patients with a history of stroke. Both patients had hemiparesis due to extensive cerebral infarction of the middle cerebral artery territory. One had paroxysmal atrial fibrillation and sick sinus syndrome, and the other had cardiac dysfunction with an ejection fraction of $35 \%$. Therefore, we attributed the cerebral infarction to embolism from a cardiogenic source in both patients. Neither of these patients had metabolic syndrome. 
Table 1 Clinical characteristics and risk-factor profiles for stroke in 77 patients with myotonic dystrophy type 1 , including 2 patients with stroke.

\begin{tabular}{|c|c|c|c|}
\hline Patient Characteristics & 77 patients & Patient 1 & Patient 2 \\
\hline \multicolumn{4}{|c|}{ Nonmodifiable risk factors } \\
\hline Age $(m \pm S D)$ & $51 \pm 14.9$ & 60 & 65 \\
\hline Male (\%) & 58 & Male & Male \\
\hline Female (\%) & 42 & - & - \\
\hline $\begin{array}{l}\text { Disease duration in years ( } \mathrm{m} \\
\qquad \pm \mathrm{SD})\end{array}$ & $15 \pm 12.1$ & 11 & 14 \\
\hline \multicolumn{4}{|c|}{ Well-documented or modifiable risk factors } \\
\hline Central obesity (\%) & 16 & - & - \\
\hline Cigarette smoking (\%) & 12 & - & - \\
\hline Alcohol consumption (\%) & 16 & - & - \\
\hline Hypertension (\%) & 11 & - & - \\
\hline Dyslipidemia (\%) & 34 & - & + \\
\hline Hypertriglyceridemia (\%) & 30 & - & + \\
\hline Low HDL (\%) & 12 & - & - \\
\hline Diabetes mellitus (\%) & 21 & - & - \\
\hline Arrhythmia (\%) & 61 & + & - \\
\hline Atrial fibrillation (\%) & 14 & + & - \\
\hline Conduction defects (\%) & 12 & - & - \\
\hline Atrial flutter (\%) & 6.5 & - & - \\
\hline High D-dimer (\%) & 13 & - & - \\
\hline \multicolumn{4}{|c|}{ Laboratory examinations } \\
\hline Arteriosclerosis on MRA (\%) & 7.9 & - & - \\
\hline $\begin{array}{l}\text { Abnormal echo images of } \\
\text { carotid artery (\%) }\end{array}$ & 25 & - & - \\
\hline Abnormal echocardiogram (\%) & 37 & + & + \\
\hline Pacemaker implantation (\%) & 5.2 & + & - \\
\hline CTG repeat numbers $(n=50)$ & $814 \pm 509$ & 1000 & 1500 \\
\hline Metabolic syndrome (\%) & 11 & - & - \\
\hline Stroke $(\%),(n=2)$ & 2.6 & + & + \\
\hline \multicolumn{4}{|c|}{$\begin{array}{l}\text { SD: Standard Deviation; HDL: High Density Lipoprotein; MRA: Magnetic } \\
\text { Resonance Angiography. }\end{array}$} \\
\hline
\end{tabular}

\section{Patient history}

Patient 1 was a 60-year-old man. He noticed slowly progressive distal-dominant muscle weakness of all four limbs and grip myotonia at the age of 43 years. He visited our hospital at the age of 49 years. Electromyography revealed myotonic discharge with myogenic changes. Genetic testing focusing on DM1 showed a CTG expansion of 1000 trinucleotides on one allele of the DMPK gene, confirming the diagnosis of DM1. At 54 years of age, the patient had an acute onset of right limb weakness and speech disturbance. The acute onset of focal neurological symptoms suggested stroke, and he was immediately transferred to our hospital. Neurological examinations revealed global aphagia and right hemiplegia. The National Institutes of Health Stroke Scale (NIHSS) score was 17. Brain MRI showed acute watershed infarction of the left Middle Cerebral Artery (MCA) territory (Figure 1). The patient received a diagnosis of paroxysmal atrial (c) Under License of Creative Commons Attribution 3.0 License fibrillation and sick sinus syndrome and underwent pacemaker implantation. He did not have diabetes, dyslipidemia, or hypertension, but had bilateral cataracts, hypogonadism, and baldness. His older sister and his uncle both had DM1, but neither had a history of stroke.

Patient 2 was a 65-year-old man. He noticed slowly progressive distal-dominant muscle weakness of all four limbs and grip myotonia at the age of 40 years. He visited our hospital and showed myotonic discharge with myogenic changes on electromyography at the age of 45 years. Genetic testing focusing on DM1 revealed a CTG expansion of 1500 trinucleotides on one allele of the DMPK gene, confirming the diagnosis of DM1. At age 59 years of age, he had an acute onset of dysarthria and left hemiparesis. The NIHSS score was 10 . Brain MRI showed acute small scattered infarcts in the right MCA territory (Figure 2). However, magnetic resonance angiography and carotid artery ultrasonography revealed no evidence of atherosclerosis.

Two-dimensional echocardiography showed a left ventricular ejection fraction of $35 \%$ and enlargement of both ventricles and the left atrium, suggesting acute heart failure. He had bilateral cataracts, hypogonadism, and baldness. Dyslipidemia was present, without diabetes or hypertension. His older and younger brothers were known to have DM1, but neither had a history of stroke.

\section{Genetic analysis}

We performed genetic examinations focusing on DM1 in 50 patients (65\%). The numbers of CTG repeats on the DMPK gene ranged from 100 to 2300 in our series (Figure 3). Two patients

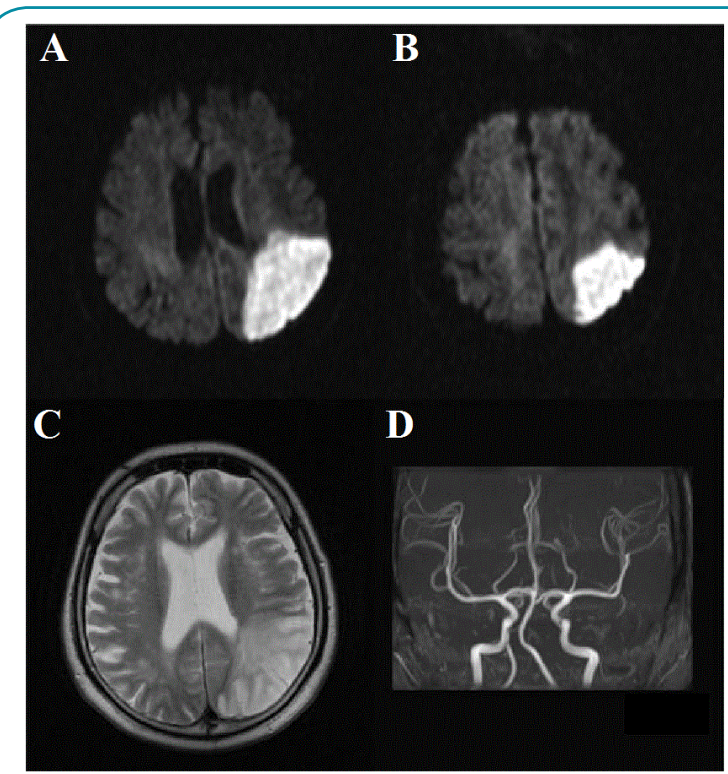

Figure 1 Magnetic resonance images of Patient 1 . Diffusionweighted images showed high intensity in the watershed area of the left middle cerebral artery and posterior cerebral artery territories at admission (A, B). Follow-up T2-weighted images 1 day after the stroke showed high intensity in the same territories (C). Magnetic resonance angiography revealed no abnormalities (D). 
with a history of stroke had highly expanded numbers of CTG repeats: 1000 in Patient 1 and 1500 in Patient 2. There was no correlation between age and the number of CTG repeats in our subjects.

\section{Discussion}

Our enrolled 77 Japanese patients with DM1 had variable frequencies of well-documented or modifiable risk-factor

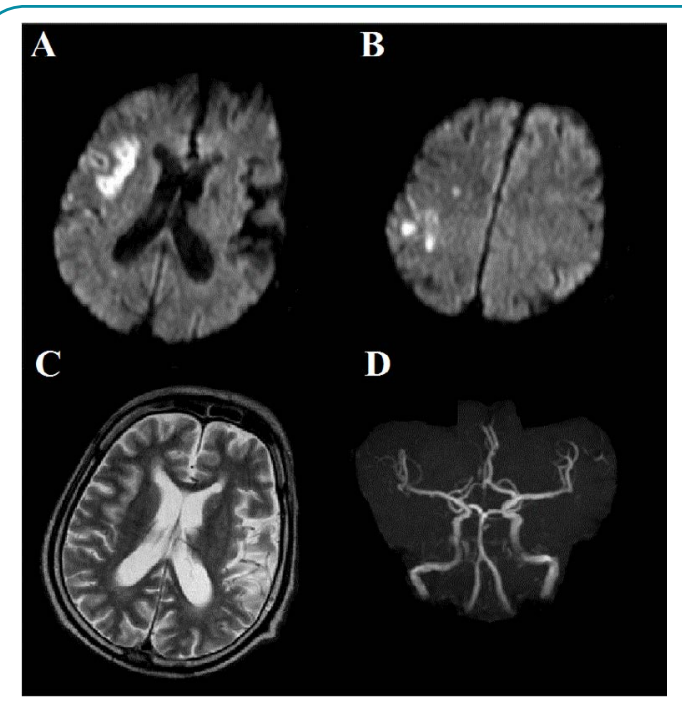

Figure 2 Magnetic resonance images of Patient 2. Diffusionweighted images showed multiple scattered, highintensity infarcts in the right middle cerebral artery territory at admission (A, B). Follow-up T2-weighted images 1 day after the stroke showed high intensity in the same territory (C). Magnetic resonance angiography revealed no abnormalities (D).

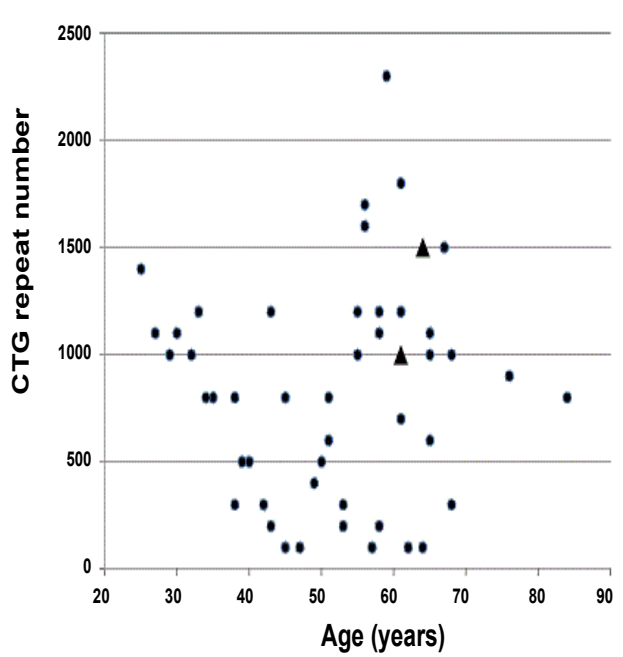

Figure 3

Scatter-plots of the numbers of CTG repeats of the DMPK gene. The numbers of CTG repeats on the DMPK gene ranged from 100 to 2300 in our patients. Two patients (triangle) with a history of stroke showed highly expanded numbers of CTG repeats (1000 and 1500). There was no correlation between age and the numbers of CTG repeats in our subjects. profiles associated with stroke. Importantly, cardiac involvement including arrhythmias was the most frequent and the most clinically significant risk factor in our patients. Subsequently, dyslipidemia and diabetes mellitus, which have been established as well-documented risk factors, also became very important factors in patients with DM1. Modifiable factors linked to lifestyle, such as cigarette smoking and alcohol consumption, were not among the most prevalent risk factors. To our knowledge, this is the first study to report the results of a comprehensive analysis of risk-factor profiles for stroke in patients with DM1.

Moreover, we identified 2 patients (2.6\%) with ischemic stroke due to cardiogenic embolism and analyzed the incidence of stroke among the DM1 patients in our study. However, the pathomechanism of stroke in patients with DM1 has not been well established or assessed. A previous study reported that the incidence of cerebral infarction in patients with DM1 was $1.6 \%$ in the US [9]. Because stroke is a relatively rare but severe complication of DM1, it is very important to manage risk factors for stroke to prevent its onset. In this connection, the stroke incidence is estimated to be $0.4 \%$ for the general population in Japan, similar to that in the US and the EU [10]. Now the distribution of risk factors for stroke in the Japanese population are also similar to those in the US and the EU, with the exception of dyslipidemia.

Importantly, we identified 2 patients who had a history of stroke caused by cardiogenic embolism. One patient had paroxysmal atrial fibrillation and sick sinus syndrome, and the other had cardiac dysfunction with an ejection fraction of $35 \%$. All previously reported patients had cardioembolic infarction [9-12]. Because many patients with DM1 have arrhythmias and cardiac dysfunction, cardiac involvement is the most important life-threatening, prognostic factor in DM1 and accounts for approximately one third of the deaths in this population [1315]. We believe that the strict management of cardiac function leads to the prevention of stroke. Patients with DM1 should therefore undergo screening for cardiac complications and risk stratification.

Previously, the incidence of ischemic stroke was reported to be $0.75 \%$ in patients with Duchenne's muscular dystrophy [16]. All of the patients with stroke had cardiomyopathy with atrial fibrillation. In addition, a young patient with limb girdle muscular dystrophy $1 \mathrm{~B}$ caused by the lamin $\mathrm{A} / \mathrm{C}$ gene was reported to have had cardioembolic stroke with atrial fibrillation [17]. Review articles estimated that the prevalence of atrial fibrillation or atrial flutter in patients with primary myopathies was $15 \%[18,19]$. The stroke rate in patients with myopathy and atrial fibrillation/ atrial flutter was around $6.5 \%$. However, we must not overlook deterioration of muscle weakness due to stroke, because patients with primary myopathies including DM1 had muscle weakness before stroke.

Moreover, we identified 8 patients (11\%) with metabolic syndrome. The most common component in patients with DM1 was hypertriglyceridemia (26\%), with hypertension (11\%) being the rarest component. The frequency of patients with metabolic syndrome is far lower than that in the general population [20]. The prevalence of metabolic syndrome is age-dependent in the 
general population. In addition, the most common component in the general population is hypertension, whereas hypertension was the rarest component in our series. This finding suggests that increased blood pressure may play a crucial role in the development of metabolic syndrome. Recently, Vujnic et al. have reported that metabolic syndrome was diagnosed in $17 \%$ of 66 Serbian patients with DM1 [21]. However, in Serbian patients the most common component was hypertriglyceridemia (66\%), followed by low HDL levels (35\%), hypertension (18\%), and central obesity $(14 \%)$, with hyperglycemia (9\%) being the rarest component. We believe that the different frequencies of risk factors between Serbian patients and Japanese patients may be related to environmental factors, dietary habits, and ethnic differences.

The numbers of CTG repeats on the DMPK gene ranged from 100 to 2300 in our series. The 2 patients with a history of stroke showed highly expanded numbers of CTG repeats (1000 and 1500). Previous studies reported that the severity of disease in DM1 may correlate with the numbers of CTG repeats $[1,5]$. However, because the number of patients with DM1 who have stroke is very small, further studies in larger study groups are needed to define genetic effects on the incidence of stroke in patients with DM1.

The main limitations of our study were a relatively small sample size and the lack of a control group and data from the general Japanese population. Because DM1 is an especially rare neuromuscular disorder, analyses based on a single-center study usually have significant statistical limitations. To provide more meaningful information for Japanese patients with DM1 and to overcome the aforementioned limitations, we are now considering a further study with participation from centers located throughout Japan.

In conclusion, we comprehensively analyzed the frequencies of well-documented or modifiable risk factors for stroke in 77
Japanese patients with DM1 and identified 2 patients (2.6\%) who had cardiogenic embolism. Stroke is a relatively rare, but severe complication of DM1. To prevent stroke, it is essential to manage cardiac involvement and arrhythmias, especially atrial fibrillation.

\section{Acknowledgement}

We thank Ms Harumi Ikeda for her technical assistance.

\section{Consent}

All patients gave fully informed consent before participation. This study was approved by the Ethics Committee of Nara Medical University School of Medicine.

\section{Availability of Data and Materials}

We do not wish to share our dataset supporting the conclusions of this article. The reason is because we did not obtain permission of our patients to provide our dataset to other researchers in the world.

\section{Competing Interests}

The authors declare that they have no competing interests.

\section{Authors' Contributions}

This systematic review was conceived by Miho Sugie, Kazuma Sugie, Nobuyuki Eura, Naoki Iwasa, Tomo Shiota, Hitoki Nanaura, Tesseki Izumi, Satoshi Ueno. Miho Sugie and Kazuma Sugie took the lead in writing this review. Nobuyuki Eura, Naoki Iwasa, Tomo Shiota, Hitoki Nanaura, Tesseki Izumi and Satoshi Ueno contributed to running the study and interpreted the data. Miho Sugie and Kazuma Sugie authored the manuscript, while the other authors read and commented on previous drafts. All authors have read and approved the final manuscript. 


\section{References}

1 Turner C, Hilton-Jones D (2010) The myotonic dystrophies: diagnosis and management. J Neurol Neurosurg Psychiatry 81: 358-367.

2 Bachmann G, Damian MS, Koch M, Schilling G, Fach B, et al. (1996) The clinical and genetic correlates of MRI findings in myotonic dystrophy. Neuroradiology 38: 629-635.

3 Harper PS, Monckton DG (2004) Myotonic dystrophy. Myology, Vol. 2 (3rd edn.) McGraw-Hill, New York, USA, pp: 1039-1076.

4 Ashizawa T, Epstein HF (1991) Ethnic distribution of myotonic dystrophy gene. Lancet 338: 642-643.

5 Sugie K, Sugie M, Taoka T, Tonomura Y, Kumazawa A, et al (2015) Characteristic MRI findings of upper limb muscle involvement in myotonic dystrophy type 1. PLoS One 10: e0125051.

6 Brook JD, McCurrach ME, Harley HG, Buckler AJ, Church D, et al. (1992) Molecular basis of myotonic dystrophy: expansion of a trinucleotide (CTG) repeat at the 3 end of a transcript encoding a protein kinase family member. Cell 68: 799-808.

7 Jauch EC, Saver JL, Adams HP Jr, Bruno A, Connors JJ, et al. (2013) Guidelines for the early management of patients with acute ischemic stroke: a guideline for healthcare professionals from the American Heart Association/American Stroke Association. Stroke 44: 870-947.

8 Alberti KG, Eckel RH, Grundy SM, Zimmet PZ, Cleeman JI, et al. (2009) Harmonizing the metabolic syndrome: a joint interim statement of the International Diabetes Federation Task Force on Epidemiology and Prevention; National Heart, Lung, and Blood Institute; American Heart Association; World Heart Federation; International Atherosclerosis Society; and International Association for the Study of Obesity. Circulation 120: 1640-1645.

9 Biller J, lonasescu V, Zellweger H, Adams HP Jr, Schultz DT (1987) Frequency of cerebral infarction in patients with inherited neuromuscular diseases. Stroke 18: 805-807.
10 Suzuki K, Sarti C, Tuomilehto J, Kutsuzowa T, Narva EV, et al. (1994) Stroke incidence and case fatality in Finland and in Akita, Japan: a comparative study. Neuroepidemiology 13: 236-44.

11 Morris LK, Cuetter AC, Gunderson CH (1982) Myotonic dystrophy, mitral valve prolapse, and cerebral embolism. Stroke 13: 93-94.

12 Cook AW, Bird TD, Spence AM, Pagon RA, Wallace JF (1978) Myotonic dystrophy, mitral-valve prolapse, and stroke. Lancet 1: 335-336.

13 Groh WJ, Groh MR, Saha C, Kincaid JC, Simmons Z, et al. (2008) Electrocardiographic abnormalities and sudden death in myotonic dystrophy type 1. N Engl J Med 358: 2688-2697.

14 Lau JK, Sy RW, Corbett A, Kritharides L (2015) Myotonic dystrophy and the heart: A systematic review of evaluation and management. Int J Cardiol 184: 600-608.

15 Mathieu J, Allard P, Potvin L, Prévost C, Bégin P (1999) A 10-year study of mortality in a cohort of patients with myotonic dystrophy. Neurology 52: 1658-1662.

16 Hanajima R, Kawai M (1996) Incidence of cerebral infarction in Duchenne muscular dystrophy. Muscle Nerve 19: 928.

17 Chen CH, Tang SC, Su YN, Yang CC, Jeng JS (2013) Cardioembolic stroke related to limb-girdle muscular dystrophy 1B. BMC Res Notes 6: 32.

18 Wahbi K, Sebag FA, Lellouche N, Lazarus A, Bécane HM, et al. (2016) Atrial flutter in myotonic dystrophy type 1: Patient characteristics and clinical outcome. Neuromuscul Disord 26: 227-233.

19 Finsterer J, Stöllberger C (2008) Atrial fibrillation/flutter in myopathies. Int J Cardiol 128: 304-310.

20 Eckel RH, Alberti KG, Grundy SM, Zimmet PZ (2010) The metabolic syndrome. Lancet 375: 181-183.

21 Vujnic M, Peric S, Popovic S, Raseta N, Ralic V, et al. (2015) Metabolic syndrome in patients with myotonic dystrophy type 1 . Muscle Nerve 52: 273-277. 